\title{
Effects of Multi-Species Direct-Fed Microbial Products on Ruminal Metatranscriptome and Carboxyl-Metabolome of Beef Steers
}

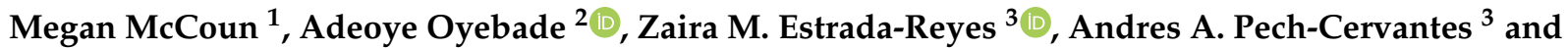 \\ Ibukun M. Ogunade ${ }^{4, *(1)}$ \\ 1 Division of Food and Animal Science, Kentucky State University, Frankfort, KY 40601, USA; \\ xtiemira@gmail.com \\ 2 Department of Animal Sciences, University of Florida, Gainesville, FL 32611, USA; adeoye.oyebade@ufl.edu \\ 3 College of Agricultural, Family Sciences, and Technology, Fort Valley State University, Fort Valley, \\ GA 31030, USA; zaira.estradareyes@fvsu.edu (Z.M.E.-R.); andres.pechcervantes@fvsu.edu (A.A.P.-C.) \\ 4 Division of Animal and Nutritional Science, West Virginia University, Morgantown, WV 26506, USA \\ * Correspondence: Ibukun.ogunade@mail.wvu.edu
}

check for updates

Citation: McCoun, M.; Oyebade, A.; Estrada-Reyes, Z.M.; Pech-Cervantes, A.A.; Ogunade, I.M. Effects of Multi-Species Direct-Fed Microbial Products on Ruminal Metatranscriptome and Carboxyl-Metabolome of Beef Steers. Animals 2021, 11, 72. https:// doi.org/10.3390/ani11010072

Received: 10 October 2020 Accepted: 18 December 2020 Published: 2 January 2021

Publisher's Note: MDPI stays neutral with regard to jurisdictional clai$\mathrm{ms}$ in published maps and institutional affiliations.

Copyright: (C) 2021 by the authors. Licensee MDPI, Basel, Switzerland. This article is an open access article distributed under the terms and conditions of the Creative Commons Attribution (CC BY) license (https:// creativecommons.org/licenses/by/ $4.0 /)$.
Simple Summary: This study examined the effects of two direct-fed microbial (DFM) products containing multiple microbial species and their fermentation products on ruminal metatranscriptome and carboxyl-metabolome of beef steers. The two DFM products altered the relative concentrations of short-chain fatty acids. No effects were detected on the functional attributes of the rumen microbiota; however, one of the two DFM products reduced the relative concentrations of metabolites involved in fatty acid peroxidation and amino acid degradation. This study demonstrated that dietary supplementation with either PROB or SYNB altered the ruminal fermentation pattern. In addition, supplemental PROB reduced the relative concentrations of metabolic products of fatty acid peroxidation and amino acid degradation.

Abstract: We examined the effects of two direct-fed microbial (DFM) products containing multiple microbial species and their fermentation products on ruminal metatranscriptome and carboxylmetabolome of beef steers. Nine ruminally-cannulated Holstein steers were assigned to 3 treatments arranged in a $3 \times 3$ Latin square design with three 21 -d periods. Dietary treatments were (1) Control (CON; basal diet without additive), (2) Commence (PROB; basal diet plus $19 \mathrm{~g} / \mathrm{d}$ of Commence), and (3) RX3 (SYNB; basal diet plus $28 \mathrm{~g} / \mathrm{d}$ of RX3). Commence and RX3 are both S. cerevisiaebased DFM products containing several microbial species and their fermentation products. Mixed ruminal contents collected multiple times after feeding on day 21 were used for metatranscriptome and carboxyl-metabolome analysis. Partial least squares discriminant analysis revealed a distinct transcriptionally active taxonomy profiles between CON and each of the PROB and SYNB samples. Compared to CON, the steers fed supplemental PROB had 3 differential (LDA $\geq 2.0 ; p \leq 0.05$ ) transcriptionally active taxa, none of which were at the species level, and those fed SYNB had eight differential (LDA $>2.0, p \leq 0.05)$ transcriptionally active taxa, but there was no difference $(p>0.05)$ between PROB and SYNB. No functional microbial genes were differentially expressed among the treatments. Compared with CON, 3 metabolites (hydroxylpropionic acid and 2 isomers of propionic acid) were increased (FC $\geq 1.2$, FDR $\leq 0.05$ ), whereas 15 metabolites, including succinic acid and fatty acid peroxidation and amino acid degradation products were reduced (FC $\leq 0.83, \mathrm{FDR} \leq 0.05$ ) by supplemental PROB. Compared with CON, 2 metabolites ( 2 isomers of propionic acid) were increased ( $F C \geq 1.2, F D R \leq 0.05$ ), whereas 2 metabolites (succinic acid and pimelate) were reduced $(F C \leq 0.83, F D R \leq 0.05)$ by supplemental SYNB. Compared to SYNB, supplemental PROB reduced (FC $\leq 0.83, F D R \leq 0.05)$ the relative abundance of four fatty acid peroxidation products in the rumen. This study demonstrated that dietary supplementation with either PROB or SYNB altered the ruminal fermentation pattern. In addition, supplemental PROB reduced concentrations of metabolic products of fatty acid peroxidation and amino acid degradation. Future studies are needed to evaluate the significance of these alterations to ruminal fatty acid and amino acid metabolisms, and their influence on beef cattle performance. 
Keywords: fatty acid peroxidation; metabolome; lactic acid bacteria; rumen fluid; Saccharomyces cerevisiae

\section{Introduction}

Direct-fed microbials (DFM) are fed as a source of active and naturally occurring microorganisms in livestock production systems to improve the health and productivity of animals [1]. The use of DFM in livestock diet is a popular practice due to public concern over the use of antibiotics in livestock production [1]. Saccharomyces cerevisiae, lactic acid-utilizing and lactic acid-producing bacteria are the most commonly used DFM in ruminant production system [2]. In recent years, most S. cerevisiae-based microbial additives are formulated to contain a combination of several microbial species or their fermentation products, in order to ensure efficacies and multi-factorial response to their supplementation [2].

Several studies utilized culture-independent molecular techniques to study the modes of action of DFM products, which include shift in rumen microbial community and fermentation pattern [3,4]. Most of the studies that evaluated a microbial community shift focused primarily on relative microbial abundance in the rumen [4,5]. However, how DFM affects the functional activity (analyzed via metatranscriptomics) of the rumen microbiome is currently unknown. Furthermore, the effects of microbial additives on an altered ruminal fermentation pattern caused by rumen microbiome shift primarily focused on ruminal short chain fatty acids, such as volatile fatty acid profile. However, little or no emphasis was placed on how DFM affects the metabolism of other carboxylic acid-containing metabolites (carboxyl-metabolome), such as fatty acid metabolites in the rumen.

Chemical isotope labeling liquid chromatography mass spectrometry (LC-MS)-based metabolomics provided an opportunity to quantitatively analyze several carboxylic acidcontaining metabolites in the biofluids [6]. Global profiling of carboxylic acid-containing metabolites in the rumen is needed, because a vast proportion of the metabolites, such as fatty acid metabolites and amino acids, in the rumen, contain at least one carboxylic acid group in their chemical structure. We had previously demonstrated that dietary supplementation of either of two multi-species DFM products improved the energy status and altered the ruminal bacterial community of beef steers [7]; however, taxonomically dissimilar microbiomes can share similar metabolic functions. Therefore, determining the effects of these DFM products on the functional attributes of the rumen microbiome and carboxyl-metabolome would enhance our understanding of their mechanisms of action. Thus, this study investigated the effects of two multi-species DFM products on ruminal metatranscriptome and carboxyl-metabolome of beef steers.

\section{Materials and Methods}

Nine ruminally cannulated Angus beef steers with a mean body weight (BW) of $243 \pm 12 \mathrm{~kg}$ were assigned to 3 treatments in a $3 \times 3$ Latin square design, with three $21-\mathrm{d}$ periods and $10-\mathrm{d}$ wash-out between periods. The steers were individually fed ( $3 \% \mathrm{BW})$ a total mixed ration containing $20.3 \%$ concentrate mix and $79.7 \%$ corn silage on a dry matter basis (Table 1), once daily at 09:00 am. Dietary treatments were (1) Control (CON; basal diet without additive), (2) Commence (PROB; basal diet plus $19 \mathrm{~g} / \mathrm{d}$ of Commence), and (3) RX3 (SYNB; basal diet plus $28 \mathrm{~g} / \mathrm{d}$ of RX3). Commence is an optimized blend of live S. cerevisiae, Enterococcus lactis, Bacillus subtilis, Enterococcus faecium, and L. casei, and their fermentation products. RX3 is an optimized blend of active S. cerevisiae and the fermentation products of S. cerevisiae, Enterococcus lactis, Bacillus licheniformis, and Bacillus subtilis. The DFM products were top-dressed daily on the total mixed ration, in the form of a premix using dried distillers grain with solubles, while a similar premix with no additive was top-dressed for the CON treatment. 
Table 1. Ingredient and chemical composition of the basal diet ${ }^{1}$.

\begin{tabular}{cc}
\hline Ingredient (\%DM) & \% of Dietary DM \\
\hline Corn silage & 79.7 \\
Dehydrated distillers grain & 9.06 \\
Soybean meal & 9.28 \\
Limestone & 0.42 \\
Deccox ${ }^{2}$ & 0.03 \\
Vitamin and mineral premix ${ }^{3}$ & 1.51 \\
& \\
DM, $\%$ & 44.5 \\
CP, \% & 14.7 \\
aNDF, \% & 38.6 \\
ADF, \% & 21.5 \\
EE, \% & 3.50 \\
Ca, \% & 0.87 \\
P, \% & 0.63 \\
TDN, \% & 72.6 \\
$\mathrm{NE}_{\mathrm{m}}, \mathrm{Mcal} / \mathrm{kg}$ & 1.72 \\
$\mathrm{NE}_{\mathrm{g}}, \mathrm{Mcal} / \mathrm{kg}$ & 1.10
\end{tabular}

${ }^{1}$ Chemical composition of complete diets calculated from analysis and concentration of individual ingredients. ${ }^{2}$ Contains $6 \%$ decoquinate for the prevention of coccidiosis (Zoetis Inc.). ${ }^{3}$ Guaranteed analysis: $15 \%$ Ca; $7.5 \%$ P; $20 \%$ salt; $1 \%$ Mg; $1 \%$ K; 3600 mg $/ \mathrm{kg} \mathrm{Mn;} 12$ mg/kg Co; 1200 mg $/ \mathrm{kg} \mathrm{Cu} ; 3600$ mg $/ \mathrm{kg} \mathrm{Zn;} 27 \mathrm{mg} / \mathrm{kg} \mathrm{Se;} 60 \mathrm{mg} / \mathrm{kg}$ I; 660,000 IU $/ \mathrm{kg}$ vitamin A; $660 \mathrm{IU} / \mathrm{kg}$ vitamin $\mathrm{E}$; and 66,000 IU $/ \mathrm{kg}$ vitamin D. ${ }^{4} \mathrm{DM}=$ dry matter; $\mathrm{CP}=$ crude protein; $\mathrm{aNDF}=$ neutral detergent fiber (amylase treated); $\mathrm{ADF}=$ acid detergent fiber; $\mathrm{EE}=$ ether extract; TDN = total digestible nutrients; $\mathrm{NE}_{\mathrm{m}}=$ net energy of maintenance; and $\mathrm{NE}_{\mathrm{g}}=$ net energy of gain. The chemical composition of the top-dressed premix was not included in the nutrient analysis.

\subsection{Rumen Fluid Sampling}

On day 21 of each period, $150 \mathrm{~mL}$ of the ruminal contents were collected via the cannula at $1,3,6,9,12$, and $18 \mathrm{~h}$ after feeding. A sample of the rumen content was manually homogenized, as described by previous studies [4,8]. In brief, the ruminal contents were hand-strained through 4 layers of cheesecloth to separate the solid and liquid fractions. The samples of the solid and liquid phases for each collection time were composited for each steer and mixed 1:1 (w/w). Due to the dynamic nature of the rumen microbiota and fermentation, mixed rumen fluid samples collected multiple times within a day is needed to reduce or eliminate variation due to the time of collection. Approximately $150 \mathrm{~mL}$ of the mixed rumen contents was then mixed with $100 \mathrm{~mL}$ of RNAlater (Thermo Fisher Scientific, Waltham, MA, USA) to prevent RNA degradation, and was stored at $-80{ }^{\circ} \mathrm{C}$, until RNA extraction and sequencing were done. Another sub-sample of the mixed rumen content was strained through 4 layers of cheesecloth to separate the solid and liquid fractions, and the liquid fraction was composited for each steer and was immediately stored at $-80^{\circ} \mathrm{C}$, until the metabolomics analysis.

\subsection{Metatranscriptomics Analysis}

Approximately $0.25 \mathrm{~g}$ of each ruminal sample underwent RNA extraction using the RNeasy PowerMicrobiome Kit (Qiagen, Valencia, CA, USA), following the manufacturer's protocol. The RNeasy PowerMicrobiome kit utilizes a cell lysis protocol, which is based on the addition of each sample to a beaded tube, in combination with Lysis buffer (Solution PM1 / $\beta$ - mercaptoethanol). All RNA extracts were quantified using the Qubit RNA High Sensitivity Kit (Invitrogen, Carlsbad, CA, USA), to confirm complete DNase treatment of the RNA extracts (DNA concentration $<0.05 \mathrm{ng} / \mu \mathrm{L}$ ). Subsequently, approximately 100 ng of extracted RNA was subjected to NEBNext Ultra RNA Library Prep (New England BioLabs, Ipswich, MA, USA) double-stranded cDNA synthesis and metatranscriptome library preparation. Quality of the final library was assessed using a high sensitivity bio-analyzer chip (Agilent, Santa Clara, CA, USA). Purified metatranscriptome libraries underwent sequencing on the Illumina HiSeq4000, following a $2 \times 150 \mathrm{bp}$ index run at the 
UC Davis Genome Center. Raw read quality was assessed using the program FastQC to obtain average $Q$ scores across the read length of all R1 and R2 sequences. The program fastp was utilized to quality-filter the raw sequence data. A sliding window filtration was utilized to cut reads at a 4-base average $Q$ score of 28 or lower, reads trimmed below 90 basepairs were discarded [9]. After filtration, the reads were subjected to host, Bos taurus, cDNA subtraction using Kraken2 [10].

\subsection{CIL-LC/MS-Based Metabolomics Analysis}

Relative quantification of metabolites containing the carboxylic acid chemical group (carboxyl-metabolome) in the rumen fluid samples was done using an CIL/LC-MS-based metabolomics technique [11]. The CIL/LC-MS technique applied the use of isotope-coded p-dimethylaminophenacyl bromide as a reagent to label carboxylic acid-containing metabolites. One (1) $\mathrm{mL}$ subsample of the rumen liquid sample was centrifuged at $15,000 \times g$ for $10 \mathrm{~min}$ and the supernatant was separated for further analysis. Normalization of the sample amount was done using liquid chromatography-ultraviolet, as previously described [12]. Relative quantification of the metabolites based on peak ratio values was performed on an LC system (Palo Alto, CA, USA) connected to a Bruker Impact HD quadrupole time-of-flight MS (Billercia, MA, USA). Detailed information of the CIL-LC/MS-based metabolomics protocol was previously reported $[11,13]$. A quality control sample, prepared by mixing equal amount of a ${ }^{12} \mathrm{C}$-labeled and a ${ }^{13} \mathrm{C}$-labeled pooled sample was injected every 9 runs, to assess the performance of the instrument.

\subsection{Data and Statistical Analysis}

\subsubsection{Metatranscriptomics Analysis}

Taxonomy annotation was done using the Kraken2 software package, with an elevated confidence score threshold of 0.1 , on the filtered reads [10]. Alpha diversity rarefaction curves were created within the QIIME 1.9.1, package using an unnormalized bacterial count table [14]. Multiple rarefactions were performed on all samples using a minimum depth of 2,000,000 sequences to a maximum depth of 20,000,000 sequences, with a step size of 2,000,000 sequences for 10 iterations. Rarefactions were then collated and compared between CON and each of PROB and SYNB, based on the Chao1 metrics. Alpha diversity comparison was conducted using a two-sample $t$-test and non-parametric Monte Carlo permutations $(\mathrm{N}=999)$ within QIIME-1.9.1. Partial least squares discriminant analysis (PLS-DA) was performed using cumulative-sum scaling normalized bacterial abundance data, using the mixOmics R package [15]. The PLS-DA model was trained using a 10-fold cross validation, and this model underwent 150 iterations. Counts per million (CPM) normalized abundances of the taxonomic profiles were formatted, as described in [16]. Linear discriminant analysis Effect Size (Lefse) comparisons were made between CON and each of PROB and SYNB groups. Alpha levels of 0.05 were used for both the Kruskal-Wallis and pairwise Wilcoxon tests. LDA scores greater than 2.0 are displayed for taxonomy. The resulting taxonomic biomarkers between the CON and PROB samples were identified and then plotted as a differential feature abundance plots. Functional gene annotation and quantification of filtered sequence data were conducted using HUMAnN2 [17]. Filtered reads were first mapped against the Uniref 90 functional gene database, which were subsequently regrouped as eggNOG orthologies. Reads per kilobase counts underwent CPM normalization to account for differences in sequencing depth among samples, resulting in a final read per kilobase per million data matrix. Linear discriminant analysis effect size comparisons were used to identify differentially expressed microbial functional genes among the treatments. Alpha levels of 0.05 were used for both the Kruskal-Wallis and pairwise Wilcoxon tests. Functional genes with LDA $>2.0$ and $p$-value $\leq 0.05$ were considered to be differentially expressed. 


\subsubsection{Metabolomics Data Analysis}

The metabolomics data were analyzed using Bruker Data Analysis 4.4 (Mung and Li, 2017). The peak pairs that were present in at least $80 \%$ of samples in any treatment group were retained. The data were normalized by ratio of total useful signal. Peak pairs were identified, based on accurate mass and retention or predicted retention time matches using a labeled metabolite library (CIL Library; [18]) and a linked identity library (LI Library; [19]). All other peak pairs were unidentified. Partial least squares discriminant analysis (PLSDA) scores plot was used to visualize the differences among the treatments. Volcano plot was constructed by plotting the fold change of each metabolite against the FDR (false discovery rate) adjusted $p$-value. The fold change was calculated as Mean [20]/Mean [21], Mean(SYNB)/Mean [21], and Mean [20]/Mean(SYNB). The criterion used was FC $\geq 1.2$ or $\leq 0.83$ and FDR adjusted $p$-value $\leq 0.05$.

\section{Results}

The effects of PROB and SYNB supplementation on ruminal bacterial community analyzed via $16 \mathrm{~S}$ rRNA sequencing, volatile fatty acid and ammonia- $N$ concentrations, and energy status of the beef steers were previously reported in our companion paper [7].

\subsection{Effects of PROB and SYNB on Ruminal Metatranscriptome Profile}

Sequencing results revealed that a range of 20-82 million clusters for all samples were retained after quality filtration and read merging (average of 38.3 million clusters per sample). The nine (9) most abundant transcriptionally active taxa at the species level, which constitute $56.8 \%$ of the total microbial community, are shown in Figure 1. The relative abundance of all transcriptionally active taxa detected in all samples is shown in Supplementary Table S1. Unclassified species belonging to Prevotella $(10.3 \pm 4.0)$ and Bacteroidales (10.0 $\pm 2.9 \%)$, and Prevotella ruminicola $(8.6 \pm 4.9 \%)$ were the most abundant transcriptionally active taxa at the species level (Figure 1).

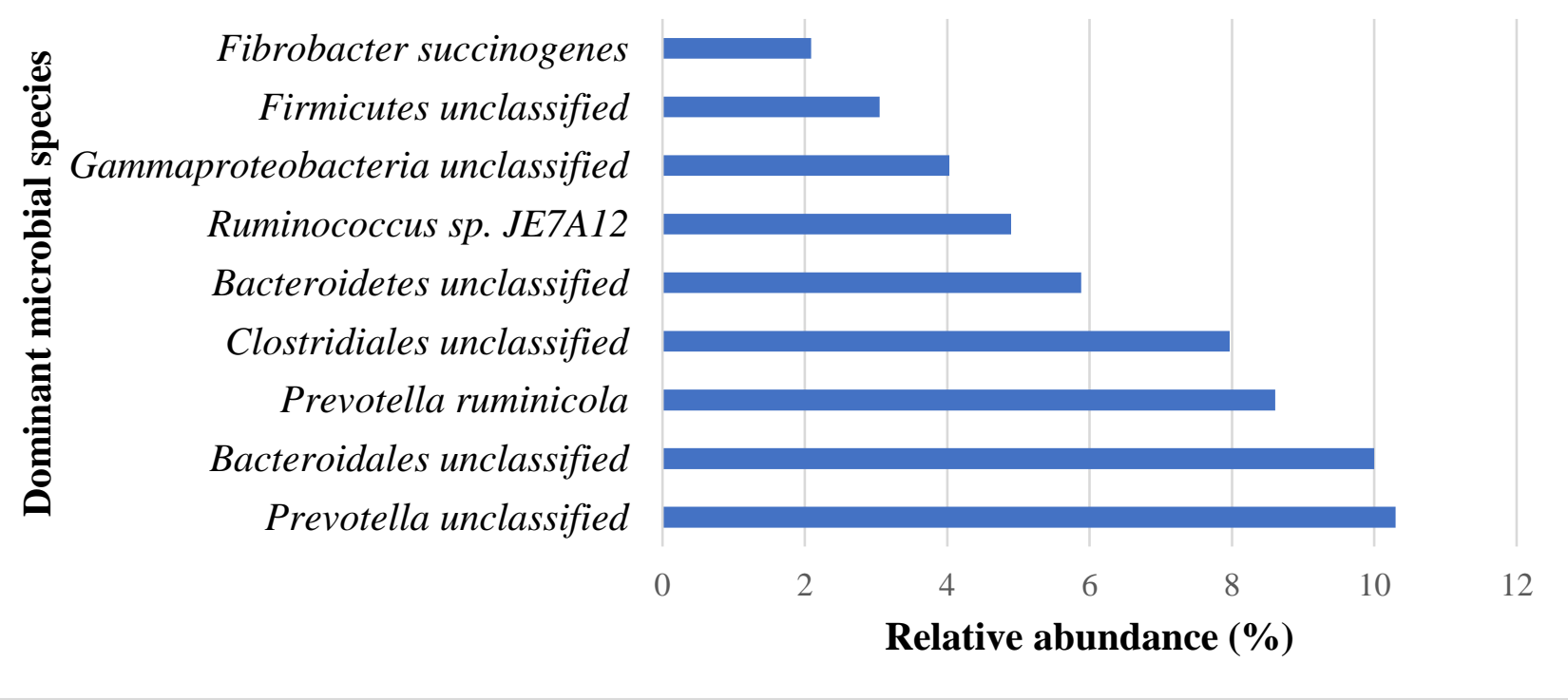

Figure 1. Relative abundance (\%) of the 9 most dominant transcriptionally active taxa at the species level.

Partial least squares discriminant analysis revealed a distinct transcriptionally active taxonomic profiles between CON and each of the PROB and SYNB samples, but there was an overlap between the PROB and SYNB (Figure 2). Linear discriminant analysis effect size analysis of the taxonomic profile revealed no differential transcriptionally active taxa between the PROB and SYNB samples (data not shown). A total of 3 differential (LDA $>2.0, p \leq 0.05)$ transcriptionally active taxa were detected between the CON and 
PROB samples, none of which were at the species level (Figure 3A). Eight differential (LDA $>2.0, p \leq 0.05$ ) transcriptionally active taxa were detected between the CON and SYNB samples (Figure 3B), two of which (Clostridium perfringens and Bifidobacterium pseudolongum) were the only differential transcriptionally active taxa at the species level and were enriched in CON compared to SYNB. Results of the functional gene annotation revealed no microbial genes were differentially expressed among the treatments (LDA $<1$; Supplementary Figure S1).

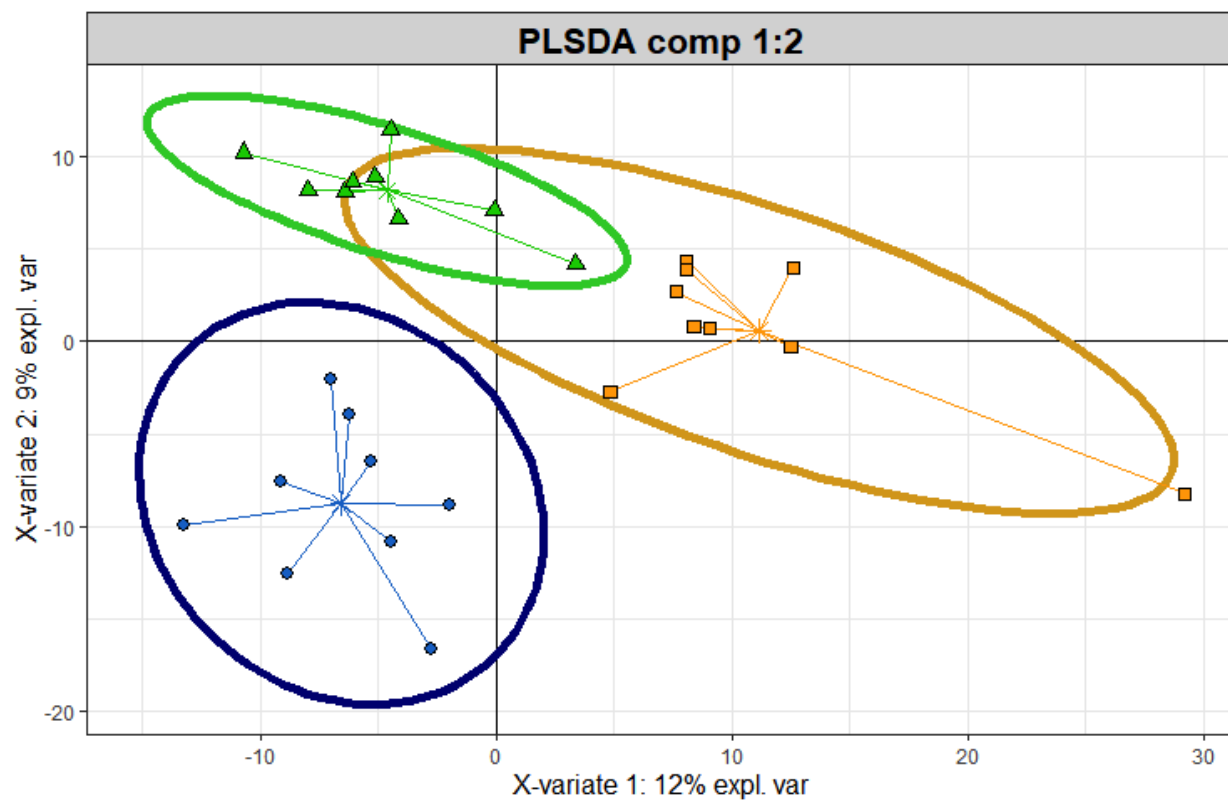

Treatment_Assignment

- CON

$\square$ PROB

$\triangle$ SYNB

Figure 2. Partial least squares discriminant analysis scores plot of the ruminal transcriptionally active microbial community of beef steers fed diet, supplemented with direct-fed microbials containing multiple microbial species and their fermentation products. $\mathrm{CON}=$ control; $\mathrm{PROB}=$ a blend of S. cerevisiae, Enterococcus lactis, Bacillus subtilis, Enterococcus faecium, and L. casei, and their fermentation products fed at $19 \mathrm{~g} /$ steer/day (PMI, Arden Hills, MN); SYNB = a blend of live S. cerevisiae and the fermentation products of S. cerevisiae, Enterococcus lactis, Bacillus licheniformis, and Bacillus subtilis fed at $28 \mathrm{~g} / \mathrm{steer} / \mathrm{day}$ PMI, Arden Hills, MN).

\subsection{Effects of PROB and SYNB on Ruminal Carboxyl-Metabolome}

An average of $2386 \pm 54$ peak pairs per sample were detected and a total number of 221 peak pairs were identified (Supplementary Table S2). Partial least squares discriminant analysis (PLS-DA) scores plot revealed separations between the metabolome of CON and each of the PROB and SYNB groups (Figure 4).

A total of 47 differential (FDR $\leq 0.05)$ peak pairs (18 of which were identified as metabolites) were detected between the CON and PROB (Figure 5A); three metabolites (hydroxylpropionic acid and 2 isomers of propionic acid) were increased (FC $\geq 1.2$, FDR $\leq 0.05$ ), whereas 15 metabolites (succinic acid, 7 isomers of 9-oxononanoic acid, 3 isomers of 12,13-epoxy-9-hydroxy-10-octadecenoate, 2-amino-5-oxohexanoate, 6-acetamido-2oxohexanoate, 8-iso prostaglandin F1alpha, and 9,10-epoxy-13-hydroxy-11-octadecenoate) were reduced $(\mathrm{FC} \leq 0.83, \mathrm{FDR} \leq 0.05)$ by supplemental PROB (Table 2 ). 


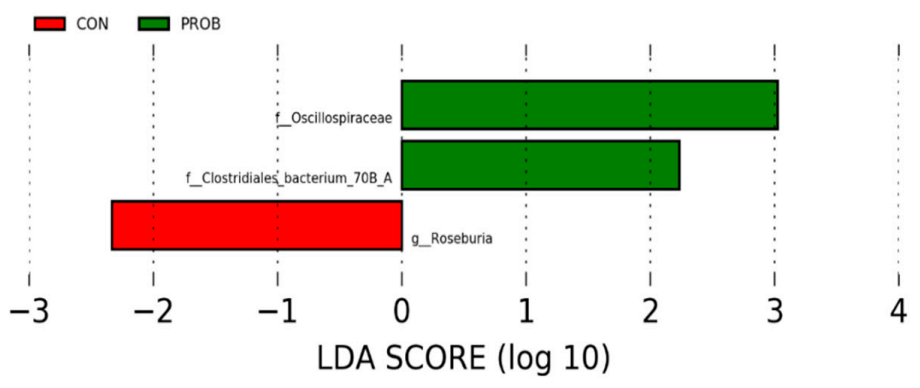

A

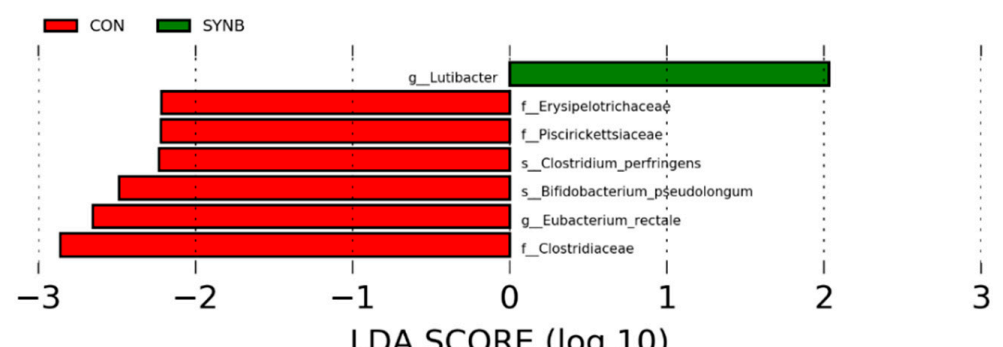

B

Figure 3. Linear discriminant analysis effect size of the ruminal transcriptionally active microbial community of beef steers fed diet supplemented with direct-fed microbials containing multiple microbial species and their fermentation products. (A) CON vs. PROB, (B) CON vs. SYNB. CON = control; $\mathrm{PROB}=$ a blend of $S$. cerevisiae, Enterococcus lactis, Bacillus subtilis, Enterococcus faecium, and L. casei, and their fermentation products fed at $19 \mathrm{~g}$ /steer/day (PMI, Arden Hills, MN); SYNB = a blend of live S. cerevisiae and the fermentation products of S. cerevisiae, Enterococcus lactis, Bacillus licheniformis, and Bacillus subtilis fed at $28 \mathrm{~g} /$ steer/day PMI, Arden Hills, MN).

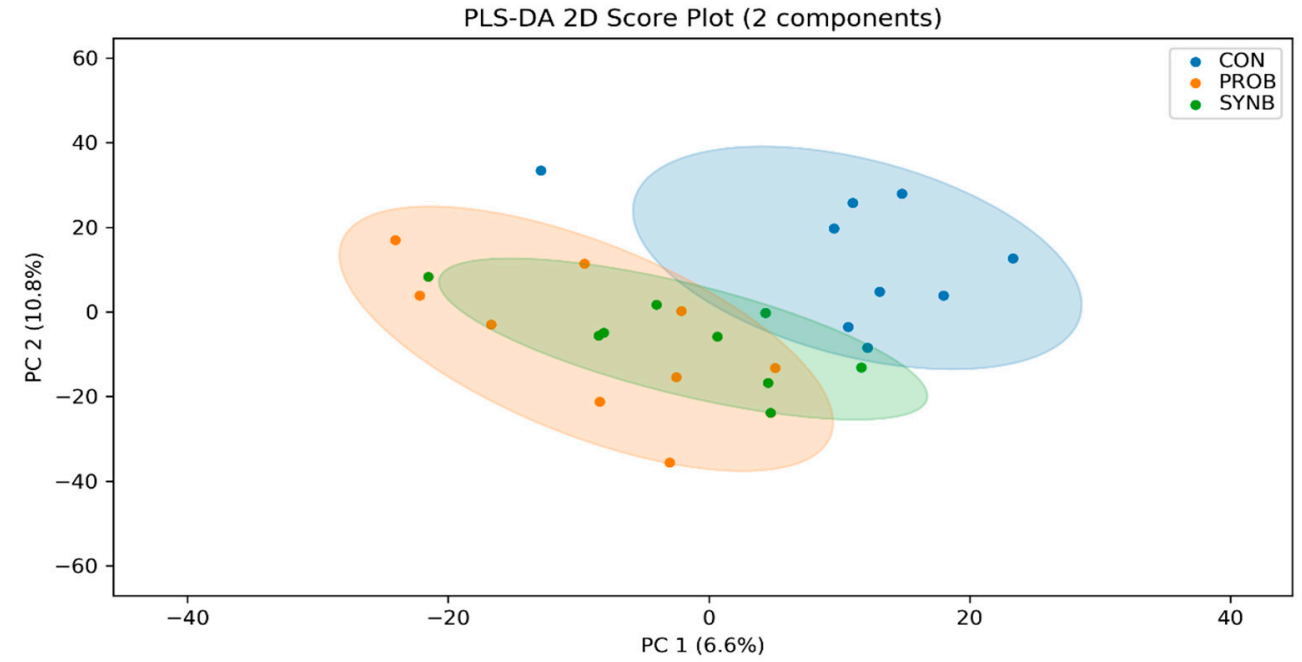

Figure 4. Partial least squares discriminant analysis (PLS-DA) scores plots of the ruminal metabolome of the beef steers fed diets supplemented with direct-fed microbials containing multiple microbial species and their fermentation products. $\mathrm{CON}=$ control; $\mathrm{PROB}=$ a blend of S. cerevisiae, Enterococcus lactis, Bacillus subtilis, Enterococcus faecium, and L. casei, and their fermentation products fed at $19 \mathrm{~g} /$ steer/day (PMI, Arden Hills, MN); SYNB = a blend of live S. cerevisiae and the fermentation products of S. cerevisiae, Enterococcus lactis, Bacillus licheniformis, and Bacillus subtilis fed at $28 \mathrm{~g} /$ steer/day PMI, Arden Hills, MN). 


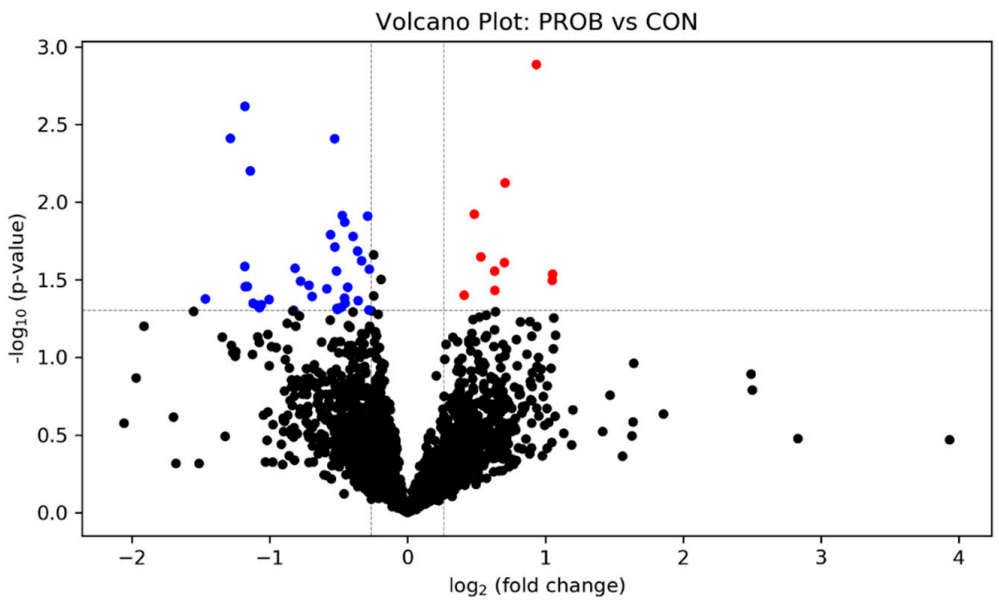

A

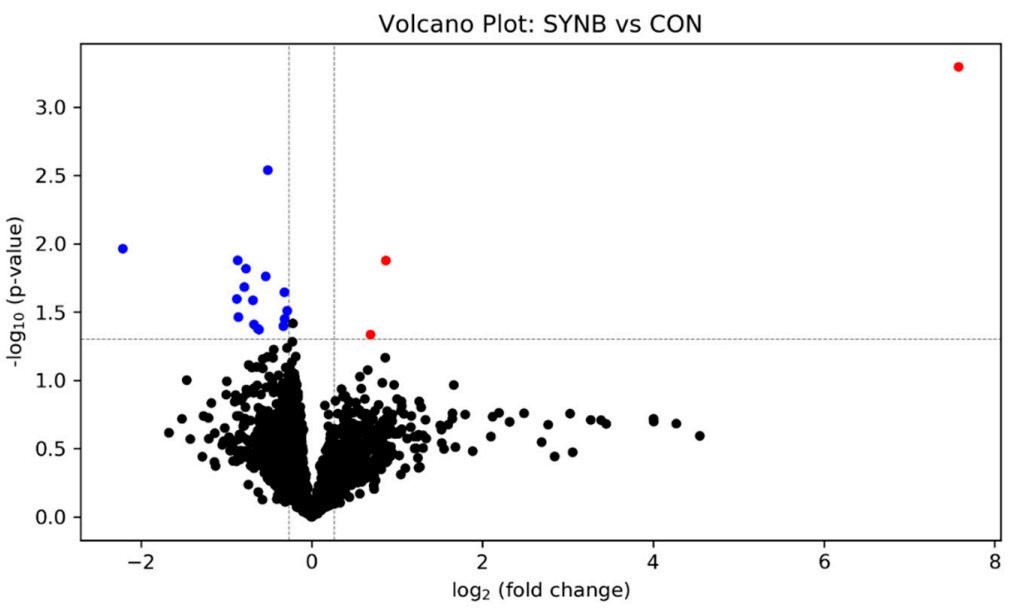

B

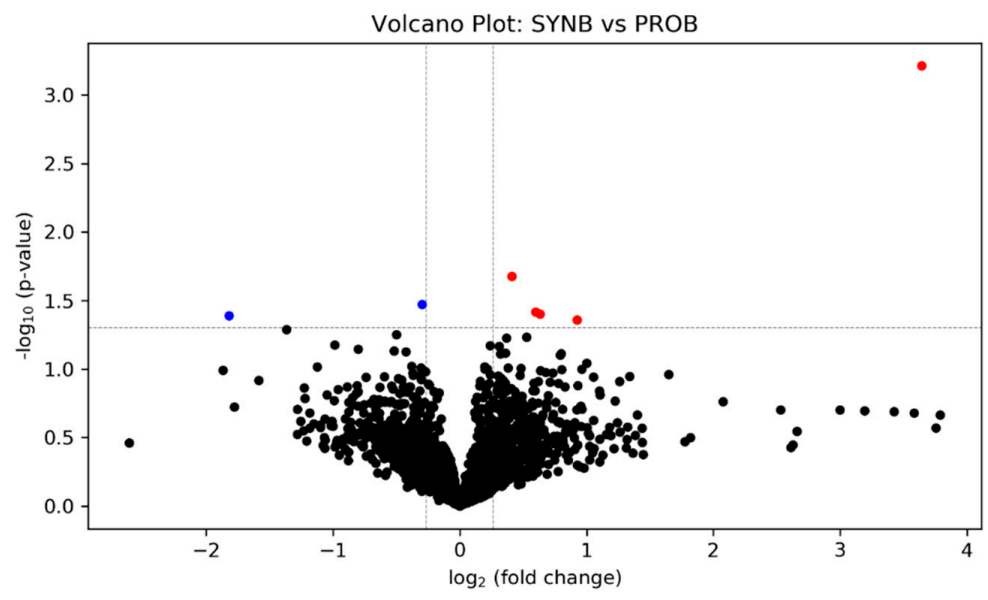

C

Figure 5. Volcano plots (A) CON vs. PROB, (B) CON vs. SYNB, and (C) PROB vs. SYNB, showing the differential ruminal metabolites in beef steers fed diets supplemented with $S$. cerevisiae-based direct-fed microbial products. FC $\geq 1.2$, p-value $\leq 0.05$ (in red)-significantly increased; FC $\leq 0.83$, $p$-value $\leq 0.05$ (in blue)—significantly reduced relative to $\mathrm{CON}$. CON = control; $\mathrm{PROB}=\mathrm{a}$ blend of S. cerevisiae, Enterococcus lactis, Bacillus subtilis, Enterococcus faecium, and L. casei, and their fermentation products fed at $19 \mathrm{~g} /$ steer/day (PMI, Arden Hills, MN); SYNB = a blend of live S. cerevisiae and the fermentation products of S. cerevisiae, Enterococcus lactis, Bacillus licheniformis, and Bacillus subtilis fed at $28 \mathrm{~g} /$ steer/day (PMI, Arden Hills, MN). 
Table 2. Identified ruminal metabolites that were altered in beef steers fed diets supplemented with direct-fed microbials containing multiple microbial species and their fermentation products.

\begin{tabular}{|c|c|c|c|}
\hline Item & Normalized RT & FC & FDR \\
\hline \multicolumn{4}{|l|}{ Effects of supplemental PROB } \\
\hline 2-amino-5-oxohexanoate & 442.4 & 0.41 & 0.01 \\
\hline 6-acetamido-2-oxohexanoate & 638.7 & 0.45 & 0.01 \\
\hline 9-oxononanoic acid & 1256.5 & 0.69 & 0.02 \\
\hline Isomer of 9-oxononanoic acid * & 1097.7 & 0.74 & 0.04 \\
\hline 8-iso prostaglandin F1alpha & 1245.5 & 0.82 & 0.01 \\
\hline 9,10-epoxy-13-hydroxy-11-octadecenoate & 1330.5 & 0.70 & 0.05 \\
\hline 12,13-epoxy-9-hydroxy-10-octadecenoate & 1337.4 & 0.70 & 0.05 \\
\hline Isomer of 12,13-epoxy-9-hydroxy-10-octadecenoate * & 1364.4 & 0.72 & 0.05 \\
\hline Succinic acid & 973.7 & 0.61 & 0.03 \\
\hline Hydroxylpropionic acid & 424.9 & 1.52 & 0.01 \\
\hline Propionic acid & 758.4 & 1.43 & 0.05 \\
\hline Isomer of propionic acid & 723.7 & 1.71 & 0.01 \\
\hline \multicolumn{4}{|l|}{ Effects of supplemental SYNB } \\
\hline $\begin{array}{l}\text { Succinic acid } \\
\end{array}$ & 973.7 & 0.74 & 0.01 \\
\hline Pimelate & 1374.2 & 0.54 & 0.03 \\
\hline Isomer of propionic acid & 723.7 & 1.32 & 0.05 \\
\hline Propionic acid & 758.4 & 1.54 & 0.02 \\
\hline \multicolumn{4}{|c|}{$\begin{array}{l}\text { Normalized RT (retention time) shows the corrected retention time of the peak pair with Universal RT Calibran } \\
\text { data. FC-fold change relative to Control; FDR-False discovery rate-adjusted p-value. }{ }^{*} \text { More than one isomer } \\
\text { of the indicated metabolite was identified. PROB = a blend of S. cerevisiae, Enterococcus lactis, Bacillus subtilis } \\
\text { Enterococcus faecium, and L. casei, and their fermentation products fed at } 19 \mathrm{~g} / \text { steer/day (PMI, Arden Hills, MN) } \\
\text { SYNB = a blend of live } S \text {. cerevisiae and the fermentation products of S. cerevisiae, Enterococcus lactis, Bacillus } \\
\text { licheniformis, and Bacillus subtilis fed at } 28 \mathrm{~g} / \text { steer } / \text { day PMI, Arden Hills, MN). Only metabolites with both } \\
\text { fold-change } \geq 1.2 \text { or } \leq 0.83 \text {, relative to control and } p \leq 0.05 \text { are shown. }\end{array}$} \\
\hline
\end{tabular}

A total of 19 differential (FDR $\leq 0.05$ ) peak pairs $(4$ of which were identified as metabolites) were detected between the CON and the SYNB groups (Figure 5B); 2 metabolites ( 2 isomers of propionic acid) were increased (FC $\geq 1.2$, FDR $\leq 0.05$ ) whereas 2 metabolites (succinic acid and pimelate) were reduced (FC $\leq 0.83$, FDR $\leq 0.05$ ) by supplemental SYNB (Table 2). When PROB and SYNB were compared, a total of 7 differential (FDR $\leq 0.05$ ) peak pairs (4 of which were identified as metabolites) were detected between the PROB and the SYNB groups (Figure 5C); the 4 metabolites (2 isomers of 9-oxononanoic acid, 12,13-epoxy9-hydroxy-10-octadecenoate, and 9,10-epoxy-13-hydroxy-11-octadecenoate) were reduced (FC $\leq 0.83$, FDR $\leq 0.05$ ) by supplemental PROB, relative to SYNB (data not shown).

\section{Discussion}

Prevotella spp. are considered the most dominant bacterial group in the rumen and were reported to grow rapidly in the presence of fermentable carbohydrates, such as those contained in corn silage [22-24]. Similar to this study, the Prevotella species were the most dominant metabolically active species in the rumen of dairy cows fed $100 \%$ corn silagebased diet [25]. Compared with CON, dietary supplementation of SYNB reduced the relative abundance of Clostridium perfringens and Bifidobacterium pseudolongum. Clostridium perfringens is a bacterial pathogen that causes intestinal disease in both animals and humans [26], while the role of Bifidobacterium pseudolongum in the rumen is not adequately described. This result is consistent with previous studies that reported the pathogen exclusion effects of DFM products against Clostridium perfringens, E. coli, and Salmonella $[27,28]$ via several mechanisms, including direct binding to the intestinal epithelium, production of compounds that are toxic to the pathogens, or competition for nutrients [2,29].

No differentially expressed microbial functional genes were detected among the treatments, indicating that the functional activity of the ruminal microbiota was not altered by the dietary supplementation of PROB or SYNB. This was in line with little or no effects on the ruminal active microbial taxonomy observed in this study. To the best of our knowledge, 
this was the first study that attempted to investigate the effects of S. cerevisiae-based DFM on the relative abundance of transcriptionally active members and functional attributes of the rumen microbiota in beef cattle that were fed a corn silage-based diet. Several previous studies that evaluated the effects of $S$. cerevisiae-based DFM on rumen microbial population, focused on the taxonomic abundance of rumen microbial population [4,30,31]. In our companion study [7], we analyzed the effects of supplemental PROB and SYNB on the relative abundance of ruminal bacterial via 16S rRNA gene sequencing and observed that, compared to CON, supplemental PROB altered the relative abundance of Succinivibrio, Rikenellaceae RC9 gut group, Succiniclasticum, Prevotella 1, and Prevotellaceae UCG-001, whereas SYNB altered the relative abundance of Succinivibrionaceae UCG-001, Succiniclasticum, Prevotella 1, and Prevotellaceae UCG-001. However, several studies demonstrated that taxonomically dissimilar microbiomes can share similar metabolic functions [21,32]. Indeed, animal phenotypes were reported to be more closely associated with rumen microbial functional profiles than taxonomic profiles [21,33]. It is important to note that we might not have captured the lowly expressed microbial gene, due to the sequencing depth of the metatranscriptomic dataset used in this study (average of 38.3 million clusters per sample). However, to date, the appropriate sequencing depth that is sufficient to capture all microbial genes in the rumen is not yet known [32].

Alteration in the concentrations of volatile fatty acids, such as increased concentration of propionic acid (and its isomers) and decreased concentration of succinic acid caused by supplemental PROB or SYNB is an indication of altered rumen fermentation pattern. Several studies demonstrated that additives containing $S$. cerevisiae altered the rumen fermentation pattern towards increased proportion of propionate, the major gluconeogenic substrate in ruminants $[34,35]$. Prevotella, the most dominant genus in the rumen, can ferment saccharides to succinate and acetate in the rumen [36]. Succinate is rapidly fermented to propionate by several ruminal microorganisms, via enzymatic processes involving coenzyme A [37]. Saccharomyces cerevisiae is capable of de novo biosynthesis of co-enzyme A, via the activities of dephospho-CoA kinase [38]. The results of our previous study [4] demonstrated that coenzyme A biosynthesis pathway was enriched in the rumen of beef steers fed diet supplemented with a $S$. cerevisiae-based additive. Thus, it is reasonable to infer that supplemental PROB and SYNB stimulated the conversion of succinate to propionate via biosynthesis of coenzyme $A$, which catalyzes the conversion of succinate to methylmalonyl-CoA and then to proponyl-CoA, which is subsequently converted to propionate [38].

The basal diet fed in this study contained over $9 \%$ dehydrated distillers grain (Table 1 ), which is known to contain high concentrations of polyunsaturated fatty acids [39]. Polyunsaturated fatty acids are very susceptible to degradation by oxygen species [40]. Up to $20 \mathrm{~L}$ of oxygen can enter the rumen of an adult ruminant daily, especially during meals, and as much as $3 \mathrm{mM}$ of dissolved oxygen can be detected in rumen fluid [41]. 9-oxononanoic acid and several epoxyols, including 12,13-epoxy-9-hydroxy-10-octadecenoate and 9,10epoxy-13-hydroxy-11-octadecenoate, are some of the major products of linoleic acid peroxidation [42]; while 8-iso-prostaglandin F $2 \alpha$ is often suggested to be an indicator of lipid peroxidation [43]. Thus, decreased concentrations of these aforementioned metabolites in steers fed supplemental PROB, compared to CON, are probably due to a reduced redox potential, possibly as a result of oxygen-scavenging activity of $S$. cerevisiae, one of the mechanisms of action by which its dietary supplementation improves rumen function $[31,44,45]$. In our previous study [4], supplementation of live yeast increased relative ruminal abundance of two microbial genes (ubiquinol-cytochrome c reductase cytochrome b subunit and cytochrome c oxidase subunit 2), which are components of the respiratory chain of $S$. cerevisiae that can catalyze the reduction of oxygen to water [46]. The fact that a similar result was not observed with supplemental SYNB, despite also containing $S$. cerevisiae, suggests that the lactic acid bacteria contained in supplemental PROB played a part. Lactic acid bacteria, including $E$. lactis and $L$. case, both of which are constituents of supplemental PROB, were demonstrated to have a high superoxide anion scavenging 
ability $[47,48]$. Lactobacillus casei was reported to reduce lipid peroxidation and improve lipid metabolism, both in blood and liver of rats [48]. Lactobacillus paracasei and other lactic acid bacteria were shown to prevent hydroxyl radical production [49]. Taken together, these results indicate that a reduced abundance of lipid peroxidation products caused by feeding supplemental PROB was either due to the antioxidant effects of lactic acid bacteria contained in supplemental PROB or a synergistic effect of both S. cerevisiae and lactic acid bacteria.

Amino acids are known to be susceptible to degradation by lipid oxidation products [50]. Histidine, and to a lesser extent, lysine are one of the most vulnerable amino acids to oxidative degradation [50,51]. Reduced concentrations of 2-amino-6-oxohexanoate and 6-acetamido-2-oxohexanoate, which are both intermediate products of lysine degradation [52], in steers fed supplemental PROB, are possibly caused by reduced ruminal concentrations of fatty acid oxidation products in these animals. This is supported by the results of our companion study, which showed a decreased ruminal ammonia-N concentration and increased ruminal concentrations of amino acids in steers fed supplemental PROB relative to CON [7]. Further studies are needed to determine how fatty acid oxidation products affect amino acid metabolism in the rumen.

\section{Conclusions}

This study demonstrated that dietary supplementation with either PROB or SYNB did not alter the functional capacity of the ruminal microbiome but altered the ruminal fermentation pattern towards increased propionate concentration. Supplemental PROB, but not SYNB, altered the ruminal carboxyl-metabolome towards reduced concentrations of metabolic products of fatty acid peroxidation and amino acid oxidative degradation. The significance of the carboxyl-metabolome alterations to ruminal fatty acid and amino acid metabolism and the influence on host performance should be explored in future studies.

Supplementary Materials: The following are available online at https:/ / www.mdpi.com/2076-261 5/11/1/72/s1. Figure S1: Linear discriminant analysis effect size comparisons of ruminal functional genes, Table S1: Relative abundance of microbial taxa, Table S2: List of peak pairs detected from CIL LC-MS measurement of the samples.

Author Contributions: I.M.O. conceived and designed the experiments. M.M. performed the experiments. I.M.O. analyzed the data. I.M.O. wrote the paper. I.M.O., A.O., A.A.P.-C., Z.M.E.-R., and M.M. revised the paper. All authors agree to be accountable for all aspects of the work. All authors have read and agreed to the published version of the manuscript.

Funding: The study was partially funded by PMI. Additional support was provided by West Virginia University Experimental Station, Morgantown, WV, USA (Scientific Article No: 3388).

Institutional Review Board Statement: The study guidelines were approved by the Institutional Animal Care and Use Committee of Kentucky State University (17-0001).

Informed Consent Statement: Not applicable.

Data Availability Statement: The data presented in this study are available on request from the corresponding author.

Conflicts of Interest: The authors declare no conflict of interest.

\section{References}

1. Krehbiel, C.R.; Rust, S.R.; Zhang, G.; Gilliland, S.E. Bacterial direct-fed microbials in ruminant diets: Performance response and mode of action. J. Anim. Sci. 2003, 81, E120-E132.

2. McAllister, T.A.; Beauchemin, K.A.; Alazzeh, A.Y.; Baah, J.; Teather, R.M.; Stanford, K. Review: The use of direct fed microbials to mitigate pathogens and enhance production in cattle. Can. J. Anim. Sci. 2011, 91, 193-211. [CrossRef]

3. Fomenky, B.E.; Do, D.N.; Talbot, G.; Chiquette, J.; Bissonnette, N.; Chouinard, Y.P.; Lessard, M.; Ibeagha-Awemu, E.M. Direct-fed microbial supplementation influences the bacteria community composition of the gastrointestinal tract of pre- and post-weaned calves. Sci. Rep. 2018, 8. [CrossRef] [PubMed]

4. Ogunade, I.M.; Lay, J.; Andries, K.; McManus, C.J.; Bebe, F. Effects of live yeast on differential genetic and functional attributes of rumen microbiota in beef cattle. J. Anim. Sci. Biotechnol. 2019, 10, 68. [CrossRef] [PubMed] 
5. Jiang, Y.; Ogunade, I.; Qi, S.; Hackmann, T.; Staples, C.; Adesogan, A. Effects of the dose and viability of Saccharomyces cerevisiae. 1. Diversity of ruminal microbes as analyzed by Illumina MiSeq sequencing and quantitative PCR. J. Dairy Sci. 2017, 100, 325-342. [CrossRef]

6. Guo, K.; Li, L. High-Performance Isotope Labeling for Profiling Carboxylic Acid-Containing Metabolites in Biofluids by Mass Spectrometry. Anal. Chem. 2010, 82, 8789-8793. [CrossRef]

7. Ogunade, I.M.; McCoun, M.; Idowu, M.D.; Peters, S.O. Comparative effects of two multispecies direct-fed microbial products on energy status, nutrient digestibility, and ruminal fermentation, bacterial community, and metabolome of beef steers. J. Anim. Sci. 2020, 98, skaa201. [CrossRef]

8. Hristov, A.N.; Callaway, T.R.; Lee, C.; Dowd, S.E. Rumen bacterial, archaeal, and fungal diversity of dairy cows in response to ingestion of lauric or myristic acid1. J. Anim. Sci. 2012, 90, 4449-4457. [CrossRef]

9. Bolger, A.M.; Lohse, M.; Usadel, B. Trimmomatic: A flexible trimmer for Illumina sequence data. Bioinformatics 2014, 30, 2114-2120. [CrossRef]

10. Wood, D.E.; Salzberg, S.L. Kraken: Ultrafast metagenomic sequence classification using exact alignments. Genome Biol. 2014, 15, R46. [CrossRef]

11. Zhao, S.; Dawe, M.; Guo, K.; Li, L. Development of High-Performance Chemical Isotope Labeling LC-MS for Profiling the Carbonyl Submetabolome. Anal. Chem. 2017, 89, 6758-6765. [CrossRef] [PubMed]

12. Wu, Y.; Li, L. Determination of Total Concentration of Chemically Labeled Metabolites as a Means of Metabolome Sample Normalization and Sample Loading Optimization in Mass Spectrometry-Based Metabolomics. Anal. Chem. 2012, 84, 10723-10731. [CrossRef] [PubMed]

13. Mung, D.; Li, L. Development of Chemical Isotope Labeling LC-MS for Milk Metabolomics: Comprehensive and Quantitative Profiling of the Amine/Phenol Submetabolome. Anal. Chem. 2017, 89, 4435-4443. [CrossRef] [PubMed]

14. Caporaso, J.G.; Kuczynski, J.; Stombaugh, J.; Bittinger, K.; Bushman, F.D.; Costello, E.K.; Fierer, N.; Peña, A.G.; Goodrich, J.K.; Gordon, J.I.; et al. QIIME allows analysis of high-throughput community sequencing data. Nat. Methods 2010, 7, 335-336. [CrossRef]

15. Rohart, F.; Gautier, B.; Singh, A.; Lê Cao, K.A. mixOmics: An R package for 'omics feature selection and multiple data integration. PLoS Comput. Biol. 2017, 13, e1005752. [CrossRef]

16. Segata, N.; Izard, J.; Waldron, L.; Gevers, D.; Miropolsky, L.; Garrett, W.S.; Huttenhower, C. Metagenomic biomarker discovery and explanation. Genome Biol. 2011, 12, R60. [CrossRef]

17. Franzosa, E.A.; McIver, L.J.; Rahnavard, G.; Thompson, L.R.; Schirmer, M.; Weingart, G.; Lipson, K.S.; Knight, R.; Caporaso, J.G.; Segata, N.; et al. Species-level functional profiling of metagenomes and metatranscriptomes. Nat. Methods 2018, 15, 962-968. [CrossRef]

18. Huan, T.; Li, L. Quantitative Metabolome Analysis Based on Chromatographic Peak Reconstruction in Chemical Isotope Labeling Liquid Chromatography Mass Spectrometry. Anal. Chem. 2015, 87, 7011-7016. [CrossRef]

19. Li, L.; Li, R.; Zhou, J.; Zuniga, A.; Stanislaus, A.E.; Wu, Y.; Huan, T.; Zheng, J.; Shi, Y.; Wishart, D.S.; et al. MyCompoundID: Using an Evidence-Based Metabolome Library for Metabolite Identification. Anal. Chem. 2013, 85, 3401-3408. [CrossRef]

20. Gibson, G.R.; Probert, H.M.; Van Loo, J.; Rastall, R.A.; Roberfroid, M.B. Dietary modulation of the human colonic microbiota: Updating the concept of prebiotics. Nutr. Res. Rev. 2004, 17, 259-275. [CrossRef]

21. Taxis, T.M.; Wolff, S.; Gregg, S.J.; Minton, N.O.; Zhang, C.; Dai, J.; Schnabel, R.D.; Taylor, J.F.; Kerley, M.S.; Pires, J.C.; et al. The players may change but the game remains: Network analyses of ruminal microbiomes suggest taxonomic differences mask functional similarity. Nucleic Acids Res. 2015. [CrossRef] [PubMed]

22. Bekele, A.Z.; Koike, S.; Kobayashi, Y. Genetic diversity and diet specificity of ruminal Prevotella revealed by $16 \mathrm{~S}$ rRNA gene-based analysis. FEMS Microbiol. Lett. 2010, 305, 49-57. [CrossRef] [PubMed]

23. Pitta, D.W.; Pinchak, W.E.; Dowd, S.E.; Osterstock, J.; Gontcharova, V.; Youn, E.; Dorton, K.; Yoon, I.; Min, B.R.; Fulford, J.D.; et al. Rumen Bacterial Diversity Dynamics Associated with Changing from Bermudagrass Hay to Grazed Winter Wheat Diets. Microb. Ecol. 2009, 59, 511-522. [CrossRef] [PubMed]

24. Stevenson, D.M.; Weimer, P.J. Dominance of Prevotella and low abundance of classical ruminal bacterial species in the bovine rumen revealed by relative quantification real-time PCR. Appl. Microbiol. Biotechnol. 2007, 75, 165-174. [CrossRef] [PubMed]

25. Lettat, A.; Hassanat, F.; Benchaar, C. Corn silage in dairy cow diets to reduce ruminal methanogenesis: Effects on the rumen metabolically active microbial communities. J. Dairy Sci. 2013, 96, 5237-5248. [CrossRef]

26. Smedley, J.G.; Fisher, D.J.; Sayeed, S.; Chakrabarti, G.; McClane, B.A. The Enteric Toxins of Clostridium Perfringens; Springer: Berlin/Heidelberg, Germany, 2004; pp. 183-204.

27. Stephens, T.P.; Loneragan, G.H.; Karunasena, E.; Brashears, M.M. Reduction of Escherichia coli O157 and Salmonella in Feces and on Hides of Feedlot Cattle Using Various Doses of a Direct-Fed Microbial. J. Food Prot. 2007, 70, 2386-2391. [CrossRef]

28. Tabe, E.S.; Oloya, J.; Doetkott, D.K.; Bauer, M.L.; Gibbs, P.S.; Khaitsa, M.L. Comparative Effect of Direct-Fed Microbials on Fecal Shedding of Escherichia coli O157:H7 and Salmonella in Naturally Infected Feedlot Cattle. J. Food Prot. 2008, 71, 539-544. [CrossRef]

29. Callaway, T.R.; Anderson, R.C.; Edrington, T.S.; Genovese, K.J.; Bischoff, K.M.; Poole, T.L.; Jung, Y.S.; Harvey, R.B.; Nisbet, D.J. What are we doing about Escherichia coli O157:H7 in cattle? J. Anim. Sci. 2004, 82, E93-E99. [CrossRef] 
30. Chaucheyras-Durand, F.; Fonty, G. Influence of a Probiotic Yeast ( Saccharomyces cerevisiae CNCM I-1077) on Microbial Colonization and Fermentations in the Rumen of Newborn Lambs. Microb. Ecol. Health Dis. 2002, 14, 30-36. [CrossRef]

31. Newbold, C.J.; Wallace, R.J.; Mcintosh, F.M. Mode of action of the yeast Saccharomyces cerevisiae as a feed additive for ruminants Br. J. Nutr. 1996, 76, 249. [CrossRef]

32. Li, F.; Hitch, T.C.; Chen, Y.; Creevey, C.J. Comparative metagenomic and metatranscriptomic analyses reveal the breed effect on the rumen microbiome and its associations with feed efficiency in beef cattle. Microbiome 2019, 7, 6. [CrossRef] [PubMed]

33. Roehe, R.; Dewhurst, R.J.; Duthie, C.A.; Rooke, J.A.; McKain, N.; Ross, D.W.; Hyslop, J.J.; Waterhouse, A.; Freeman, T.C.; Watson, M.; et al. Bovine Host Genetic Variation Influences Rumen Microbial Methane Production with Best Selection Criterion for Low Methane Emitting and Efficiently Feed Converting Hosts Based on Metagenomic Gene Abundance. PLoS Genet. 2016, 12, e1005846. [CrossRef] [PubMed]

34. Kiran, R.R.; Kumar, D.S. Influence of yeast culture supplementation on rumen fermentation of bulls fed complete rations. Int. J. Agric. Sci. Vet. Med. 2013, 1, 8-15.

35. Mao, S.Y.; Zhang, R.Y.; Wang, D.S.; Zhut, W.Y. Impact of subacute ruminal acidosis (SARA) adaptation on rumen microbiota in dairy cattle using pyrosequencing. Anaerobe 2013, 24, 12-19. [CrossRef]

36. Ueki, A.; Akasaka, H.; Satoh, A.; Suzuki, D.; Ueki, K. Prevotella paludivivens sp. nov., a novel strictly anaerobic, Gram-negative, hemicellulose-decomposing bacterium isolated from plant residue and rice roots in irrigated rice-field soil. Int. J. Syst. Evol. Microbiol. 2007, 57, 1803-1809. [CrossRef]

37. White, W.H.; Gunyuzlu, P.L.; Toyn, J.H. Saccharomyces cerevisiaeIs Capable ofde NovoPantothenic Acid Biosynthesis Involving a Novel Pathway of $\beta$-Alanine Production from Spermine. J. Biol. Chem. 2001, 276, 10794-10800. [CrossRef]

38. Wirth, R.; Kádár, G.; Kakuk, B.; Maróti, G.; Bagi, Z.; Szilágyi, Á.; Rákhely, G.; Horváth, J.; Kovács, K.L. The planktonic core microbiome and core functions in the cattle rumen by next generation sequencing. Front. Microbiol. 2018, 9, 2285. [CrossRef]

39. Xu, L.; Jin, Y.; He, M.L.; Li, C.; Beauchemin, K.A.; Yang, W.Z. Effects of increasing levels of corn dried distillers grains with solubles and monensin on ruminal biohydrogenation and duodenal flows of fatty acids in beef heifers fed high-grain diets1. J. Anim. Sci. 2014, 92, 1089-1098. [CrossRef] 7

40. Xiao, M.; Zhong, H.; Xia, L.; Tao, Y.; Yin, H. Pathophysiology of mitochondrial lipid oxidation: Role of 4-hydroxynonenal (4-HNE) and other bioactive lipids in mitochondria. Free Radic. Biol. Med. 2017, 111, 316-327. [CrossRef]

41. Ellis, J.E.; Williams, A.G.; Lloyd, D. Oxygen consumption by ruminal microorganisms: Protozoal and bacterial contributions. Appl. Environ. Microbiol. 1989, 55, 2583-2587. [CrossRef]

42. Ren, R.; Hashimoto, T.; Mizuno, M.; Takigawa, H.; Yoshida, M.; Azuma, T.; Kanazawa, K. A lipid peroxidation product 9oxononanoic acid induces phospholipase A2 activity and thromboxane A2 production in human blood. J. Clin. Biochem. Nutr. 2013, 52, 228-233. [CrossRef] [PubMed]

43. Liang, Y.; Wei, P.; Duke, R.W.; Reaven, P.D.; Harman, S.M.; Cutler, R.G.; Heward, C.B. Quantification of 8-iso-prostaglandin-F2 $\alpha$ and 2,3-dinor-8-iso-prostaglandin-F2 $\alpha$ in human urine using liquid chromatography-tandem mass spectrometry. Free Radic. Biol. Med. 2003, 34, 409-418. [CrossRef]

44. Chaucheyras-Durand, F.; Walker, N.D.; Bach, A. Effects of active dry yeasts on the rumen microbial ecosystem: Past, present and future. Anim. Feed Sci. Technol. 2008, 145, 5-26. [CrossRef]

45. Ogunade, I.; Schweickart, H.; McCoun, M.; Cannon, K.; McManus, C. Integrating 16S rRNA sequencing and LC-MS-based metabolomics to evaluate the effects of live yeast on rumen function in beef cattle. Animals 2019, 9, 28. [CrossRef] [PubMed]

46. Beattie, D.S.; Jenkins, H.C.; Howton, M.M. Biochemical Evidence for the Orientation of Cytochrome b in the Yeast Mitochondrial Membrane in the Eight-Helix Model. Arch. Biochem. Biophys. 1994, 312, 292-300. [CrossRef] [PubMed]

47. Sharma, S.; Chaturvedi, J.; Chaudhari, B.P.; Singh, R.L.; Kakkar, P. Probiotic Enterococcus lactis IITRHR1 protects against acetaminophen-induced hepatotoxicity. Nutrition 2012, 28, 173-181. [CrossRef] [PubMed]

48. Zhang, Y.; Du, R.; Wang, L.; Zhang, H. The antioxidative effects of probiotic Lactobacillus casei Zhang on the hyperlipidemic rats. Eur. Food Res. Technol. 2010, 231, 151-158. [CrossRef]

49. Sun, J.; Hu, X.L.; Le, G.W.; Shi, Y.H. Lactobacilli prevent hydroxy radical production and inhibit Escherichia coli and Enterococcus growth in system mimicking colon fermentation. Lett. Appl. Microbiol. 2010, 50, 264-269. [CrossRef]

50. Uchida, K. Histidine and lysine as targets of oxidative modification. Amino Acids 2003, 25, 249-257. [CrossRef]

51. Requena, J.R.; Chao, C.C.; Levine, R.L.; Stadtman, E.R. Glutamic and aminoadipic semialdehydes are the main carbonyl products of metal-catalyzed oxidation of proteins. Proc. Natl. Acad. Sci. USA 2001, 98, 69-74. [CrossRef]

52. Gao, X.; Liang, M.; Fang, Y.; Zhao, F.; Tian, J.; Zhang, X.; Qin, X. Deciphering the differential effective and toxic responses of bupleuri radix following the induction of chronic unpredictable mild stress and in healthy rats based on serum metabolic profiles. Front. Pharmacol. 2018, 8. [CrossRef] [PubMed] 